\title{
Efficacy and safety of bempedoic acid alone or combining with other lipid- lowering therapies in hypercholesterolemic patients: a meta-analysis of randomized controlled trials
}

Xiang Zhao ${ }^{1,2}$, Xubiao Ma², Xing Luo ${ }^{2}$, Zhihua Shi ${ }^{2,3}$, Ziwen Deng ${ }^{2,3}$, Yuanxiang Jin ${ }^{2,3}$, Zhipeng Xiao ${ }^{2}$, Liming Tan ${ }^{4}$,

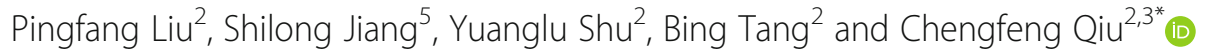

\begin{abstract}
Background: Bempedoic acid is a new drug that reduces cholesterol synthesis via inhibiting ATP citrate lyase. It remains unclear whether the combination of bempedoic acid and other lipid-lowering drugs is better than these drugs alone. This study systematically reviewed the efficacy and safety of bempedoic acid monotherapy or combination togethers in hypercholesterolemic patients.

Methods: Randomized controlled trials were searched across Medline, Embase, Cochrane library, web of science, etc. The net change scores [least squares mean (LSM) percentage change] in LDL-C level were meta-analyzed using weighted mean difference. The reductions in other lipids including total cholesterol (TC), non-high-density lipoprotein cholesterol (non-HDL-C) and apolipoprotein (ApoB) and high sensitivity C reactive protein (hsCRP) were also assessed. Odds ratio (OR) of the incidence of adverse events (AEs) were calculated to evaluate the safety of bempedoic acid.

\footnotetext{
*Correspondence: qiuchengfeng0721@163.com

Evidence-based Medicine and Clinical Center, The First People's Hospital of

Huaihua, University of South China, Huaihua 418000, People's Republic of

China

${ }^{3}$ Department of Pharmacology, The First People's Hospital of Huaihua, University of South China, Huaihua 418000, People's Republic of China

Full list of author information is available at the end of the article
}

(C) The Author(s). 2020 Open Access This article is licensed under a Creative Commons Attribution 4.0 International License, which permits use, sharing, adaptation, distribution and reproduction in any medium or format, as long as you give appropriate credit to the original author(s) and the source, provide a link to the Creative Commons licence, and indicate if changes were made. The images or other third party material in this article are included in the article's Creative Commons licence, unless indicated otherwise in a credit line to the material. If material is not included in the article's Creative Commons licence and your intended use is not permitted by statutory regulation or exceeds the permitted use, you will need to obtain permission directly from the copyright holder. To view a copy of this licence, visit http://creativecommons.org/licenses/by/4.0/. The Creative Commons Public Domain Dedication waiver (http://creativecommons.org/publicdomain/zero/1.0/) applies to the data made available in this article, unless otherwise stated in a credit line to the data. 


\begin{abstract}
(Continued from previous page)
Results: A total of 13 trials (4858 participates) were included. Pooled data showed that the combination togethers resulted in greater reductions in LDL-C level than monotherapies (bempedoic acid + statin vs. statin: LSM difference $(\%),-18.37,95 \% \mathrm{Cl},-20.16$ to $-16.57, \mathrm{I}^{2}=0$; bempedoic acid + ezetimibe vs. ezetimibe: LSM difference (\%), -18.89 , $95 \% \mathrm{Cl},-29.66$ to $-8.13, \mathrm{I}^{2}=87 \%$ ). But the difference in efficacy between bempedoic acid and ezetimibe was not obvious. Meta-regression analysis showed the treatment duration was a source of heterogeneity (adj $\mathrm{R}^{2}=16.92,95 \% \mathrm{Cl}$, 0.04 to 0.72 ). Furthermore, the background therapy of statin before screening decreased the efficacy of bempedoic acid. In addition, bempedoic acid also resulted in a significant reduction in TC, non-HDL-C, ApoB and hsCRP level. The OR of muscle-related AEs by the combination of bempedoic acid and statin was $1.29\left(95 \% \mathrm{Cl}, 1.00\right.$ to $\left.1.67, \mathrm{I}^{2}=0\right)$ when compared with statin alone.

Conclusion: This study showed the efficacy of combination togethers were similar but stronger than these drugs alone. Of note, a trend of high risk of muscle-related AEs by the combination of bempedoic acid and statin was observed, though it is not statistically significant, such risk is needed to be confirmed by more trials, because it is important for us to determine which is the better combinative administration for statin-intolerant patients.
\end{abstract}

Keywords: Bempedoic acid, Statin, Ezetimibe, Low-density lipoprotein cholesterol, Adverse events, Meta-analysis

\section{Background}

Lipid-lowering therapy mainly targeting LDL-C is an effective way to reduce cardiovascular risk [1]. Current clinical guidelines all recommend statins as the firstclass option both for primary and second prevention [2, 3]. However, even having received maximally tolerated statins, there is also a high proportion of patients hardly reach the LDL-C goal.

Statins inhibit 3-hydroxy-3-methylglutaryl-coenzyme A reductase (HMGCR) to reduce cholesterol-biosynthesis, and thus upregulate the expression of hepatic LDL receptor (LDLR), eventually lead to an increase in LDL-C clearance. Adenosine triphosphate-citrate lyase (ACL), a key enzyme that locates in the upstream of HMGCR in the cholesterol-biosynthesis pathway [4]. It implicates that ACL inhibition may present a similar effect on LDL-C reduction as HMGCR inhibition. Recently a mendelian randomization study reported that variants in $A C L Y$, the gene that encodes the ACL, was significantly associated with decreased LDL-C level, as well as decreased cardiovascular risk [5]. These findings provide substantial evidence that ACL could be a threptic target. However, genetic variants result in a lifelong inhibition of ACL, which is much different from the relative shorter-term exposure of ACL inhibitors [6]. Thus, it is necessary to assess the efficacy and safety of ACL inhibitors by using randomized controlled trials (RCTs). Bempedoic acid (ETC-1002), an oral inhibitor of ACL, converts to the active form ETC-1002-CoA by the enzyme very long-chain acyl-CoA synthetase 1 (ACSVL1) [7], which is particularly expressed in the liver but not in the peripheral tissues, including muscles. So, such liver-specific action of bempedoic acid may avoid or decrease the statin-related muscle disorders $[8,9]$.

Importantly, the clinical use of bempedoic acid is deserved to be explored. With the similar mechanism of action as statins, bempedoic acid is designed to be used in the patients with statin-intolerance. Several metaanalyses reported the efficacy of LDL-C reduction by bempedoic acid [10-12]. However, the biggest uncertainty is whether the combination togethers of bempedoic acid and other lipid-lowering drugs are better than these drugs alone. The current study systematically reviewed the efficacy and safety of bempedoic acid alone or combining with statins or ezetimibe on hypercholesterolemic patients by meta-analyses.

\section{Methods}

This study pooled the trial-level data and followed the principles recommended by the Cochrane handbook for performing and reporting intervention system review [13].

\section{Data sources and search strategy}

Studies regarding to the bempedoic acid treatment in hypercholesterolemia patients were considered as potential eligible. Two investigators (SZH and DZW) independently conducted the literature search through those following databases: Medline, Embase, Cochrane Central Register of Controlled Trials (CENTRAL), web of science and websites (www.clinicaltrials.gov). Two major ways were used to search literature. First, the following keywords ("ETC-1002" OR "bempedoic acid") AND ("randomized controlled trial" OR "controlled clinical trial" OR "trial") were used for searching through databases; second, we also performed a manual searching by scrutinizing the reference lists from all relevant articles. Literature search was updated on 11 July 2020. The details of search algorithm of Medline (Via PubMed) were provided in Additional file 1.

\section{Study selection and data extraction}

Predefined inclusion criteria for study selection was listed as follows: (i) study population included adults 
with hypercholesterolemia received bempedoic acid alone or combining with other lipid-lowering drugs; (ii) end points included efficacy outcomes (the net change scores of lipids level from baseline) and safety outcomes (the incidence of adverse events related to therapy drugs); (iii) the study was designed as clinical trials of randomized, double-blind, placebo- or active-controlled. Of note, studies that missed important information or reported the same population were excluded.

The following information was extracted from the primary text of individual study: the first author, publication year, clinical trial number, follow-up duration, the sample size of randomized patients, demographic and clinical characteristics of participates, baseline LDL-C level, least squares mean (LSM) difference of LDL-C level and the number of AEs across different intervention groups.

Two investigators (JYX and XZP) independently conducted the study selection and data extraction according to standard criteria and data sheets. All the disagreements were resolved through consulting with the third investigator $(\mathrm{QCF})$ to reach the final consensus.

\section{End points}

The efficacy of bempedoic acid was assessed using the net percentage change in LDL-C level from baseline over the follow-up duration. The other lipids including total cholesterol (TC), non-high-density lipoprotein cholesterol (non-HDL-C), apolipoprotein (ApoB) and high sensitivity $\mathrm{C}$ reactive protein (hSCRP) were also evaluated. For the overall safety assessment, the incidence of any AEs, serious AEs and muscle-related disorders were compared between different intervention groups.

\section{Bias risk assessment}

Cochrane risk of bias assessment tool was used to judge the bias risk of included trials. Two investigators (LX and JSL) independently assessed the allocation sequence generation, allocation concealment, blinding of participants and investigators, completeness of outcome data and selective outcome reporting of individual trial.

\section{Statistical analysis}

For the efficacy assessment, the net change scores which presented as LSM percentage change in lipids level and hsCRP level from baseline were pooled using the DerSimonian-Laird random-effect model. Where the LSM percentage changes of two groups were reported, the net change score was calculated using the following formula: net change score $=$ LSM percentage change of bempedoic acid group - LSM percentage change of control group [14]. Multiple interventions with different dose and different follow-up in one trial were combined to create a single pairwise comparison by using a weighted average [14]. Pooled effect size was represented as weight mean difference (WMD) of net percentage change and 95\% confidence interval (CI). In addition, random-effect meta-regression analyses with the restricted maximum likelihood estimation were performed to evaluate whether the magnitude of LDL-C reduction by bemedoic acid was associated with treatment dose and duration. Subgroup-analyses sorted by statin intensity were also conducted. To estimate the safety of bempedoic acid treatment, the incidence of AEs across groups were compared, odd ratio (ORs) with 95\% CI was used to describe the effect estimate.

Between-study heterogeneity was quantitatively calculated using the $\mathrm{I}^{2}$ index [15]. Four levels were assigned to the heterogeneity assessment according to the value of $\mathrm{I}^{2}$ index (0-25\%: no heterogeneity; 25-50\%: moderate heterogeneity; 50-75\%: large heterogeneity; and 75\%-to 100\%: extreme heterogeneity). Potential publication bias was evaluated by visual inspection of funnel plots for asymmetry [16]. Two-tailed $\alpha$ level of significance was set at 0.05 .

All statistical analyses were performed with Review Manager Version 5.3 (The Nordic Cochrane Center, Copenhagen, Denmark) and STATA/SE.12.0 (StataCorp, College station, Texas, USA).

\section{Results}

\section{Characteristics of included trials}

According to the predefined criteria, a total of 13 randomized control trials (RCTs) with 4858 participates were finally included in the current meta-analysis (Fig. 1). Six trials regarded to the combination of bempedoic acid and statins versus statins alone, two trials regarded to the combination of bempedoic acid and ezetimibe versus ezetimibe alone, two trials regarded to the combination of bempedoic acid and ezetimibe versus placebo, five trials regarded to bempedoic acid versus placebo and two trials regarded to bempedoic acid versus ezetimibe. All the participates were hypercholesterolemic patients with the baseline LDL-C level of $70 \mathrm{mg}$ per deciliter at least or more. The mean age of participates was 61 years, 61.82\% (3003) were males and $88.18 \%$ (4284) were white race. Among them, $72.05 \%$ (3500) were at high CVD risk at screening. More details of study characteristics were listed in the Table 1.

\section{Bempedoic acid significantly reduced LDL-C level}

As showed in the Fig. 2, both bempedoic acid monotherapy or combining with statin or ezetimibe were all significantly reduced LDL-C level. Of note, when compared with statin and ezetimibe alone, the combination togethers all resulted in an additional reductions in LDL-C level (bempedoic acid + statin vs. stain, LSM difference [\%]: $-18.37,95 \%$ CI: -20.16 to $-16.57, \mathrm{I}^{2}=0$; bempedoic acid 


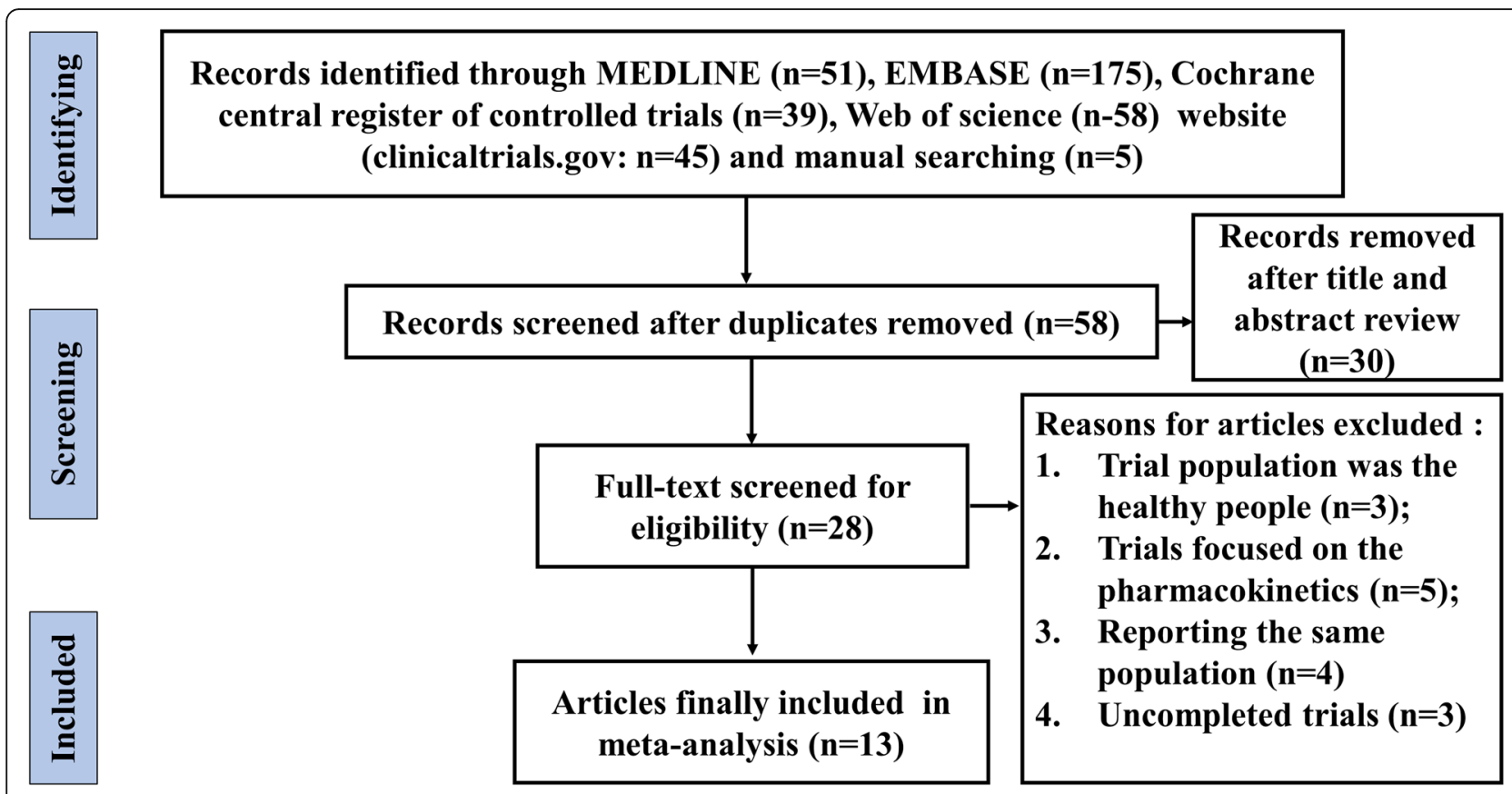

Fig. 1 Flow diagram of study screen process

+ ezetimibe vs. ezetimibe, LSM difference [\%]: -18.89, 95\% $\mathrm{CI},-29.66$ to $-8.13, \mathrm{I}^{2}=87 \%$; subgroup differences, $p=$ $0.92)$. The magnitude of LDL-C reduction by the combination together (bempedoic acid + ezetimibe) was greater than bempedoic acid alone (bempedoic acid + ezetimibe vs. placebo, LSM difference [\%]: - 37.82, 95\% CI, -41.85 to $-33.79, \mathrm{I}^{2}=0$; bempedoic acid vs. placebo, LSM difference [\%]: $-25.01,95 \% \mathrm{CI},-30.66$ to $-19.35, \mathrm{I}^{2}=86 \%$; subgroup differences, $p<0.000)$. But difference in the magnitude of LDL-C reduction between bempedoic acid and ezetimibe was not obvious.

Meta-regression analysis showed a negative association between the magnitude of LDL-C-lowering and the follow-up duration (Fig. $3 \mathrm{~A}$, adj $\mathrm{R}^{2}=16.92$, 95\% CI, 0.04 to 0.72 ). Though a trend of positive association between the LDL-C reduction and treatment dose was observed, but it was not statistically significant (Fig. $3 \mathrm{~B}$, adj $\mathrm{R}^{2}=$ 9.67, $95 \% \mathrm{CI}, 0.15$ to 0.01 ). When compared with statin alone, the net percent reduction in LDL-C by the combination of bempedoic acid and statin at week 8, 12, 24 and 52 were $-22 \%$ ( $95 \% \mathrm{CI},-32.41 \%$ to $-11.59 \%),-19.13 \%$ (95\% CI, $-20.80 \%$ to $-17.46 \%),-18.65 \%$ (95\% CI, $20.57 \%$ to $-16.73 \%$ ) and $-16.10 \%$ (95\% CI, $-18.35 \%$ to $13.35 \%$ ), respectively. Furthermore, we conducted a subgroup analysis which stratified by the background therapy of statin before screening, the magnitude of LDL-C reductions across the subgroups represented as $-28.49 \%$ (95\% CI, -40.44 to -16.54 ), $-20.02 \%$ (95\% CI, -22.71 to 17.34 ) and $-17.64 \%$ (95\% CI, -20.27 to -15.00 ) for nonusing, low or moderate and high intensity statin group, respectively, $p$ value for the differences between groups was $<0.000$ (Fig. 4).

Bempedoic acid reduced TC, non-HDL-C, ApoB and hsCRP levels

Pooled results showed that bempedoic acid also resulted in a significant reduction in TC, non-HDL-C, ApoB and hsCRP levels (Table 2). Compared with the statin alone, the combination of bempedoic acid and statin represented $-11.40 \%$ (95\% CI, -12.15 to $-10.64, \mathrm{I}^{2}=32.40 \%$ ), $13.57 \%$ (95\% CI, -14.52 to $\left.-12.62, \mathrm{I}^{2}=0\right), 12.73 \%$ (95\% CI, -14.71 to $\left.-10.75, \mathrm{I}^{2}=45.80 \%\right)$ and $-20.72 \%(95 \% \mathrm{CI}$, $\left.-29.21,-12.23, \mathrm{I}^{2}=77.8 \%\right)$ reductions in TC, non-HDL$\mathrm{C}, \mathrm{ApoB}$ and hsCRP levels, resepectively. The efficacy of bempedoic acid plus ezetimibe was similar as the bempedoic acid plus statin.

\section{Safety assessment of bempedoic acid treatment}

For the safety assessment, bempedoic acid alone or combining with statin or ezetimibe did not show a statistical difference in the incidence of any AEs, serious AEs and the AEs which lead to discontinuation of treatment (Table 3). Of note, the OR of muscle-related AEs by the combination of bempedoic acid and statin was 1.29 (95\% $\mathrm{CI}, 1.00$ to $1.67, \mathrm{I}^{2}=0$ ) when compared with statin alone.

\section{Bias assessment}

The allocation sequence generation, blinding of participants and investigators, blinding of participants and investigators and selective outcome reporting were all 


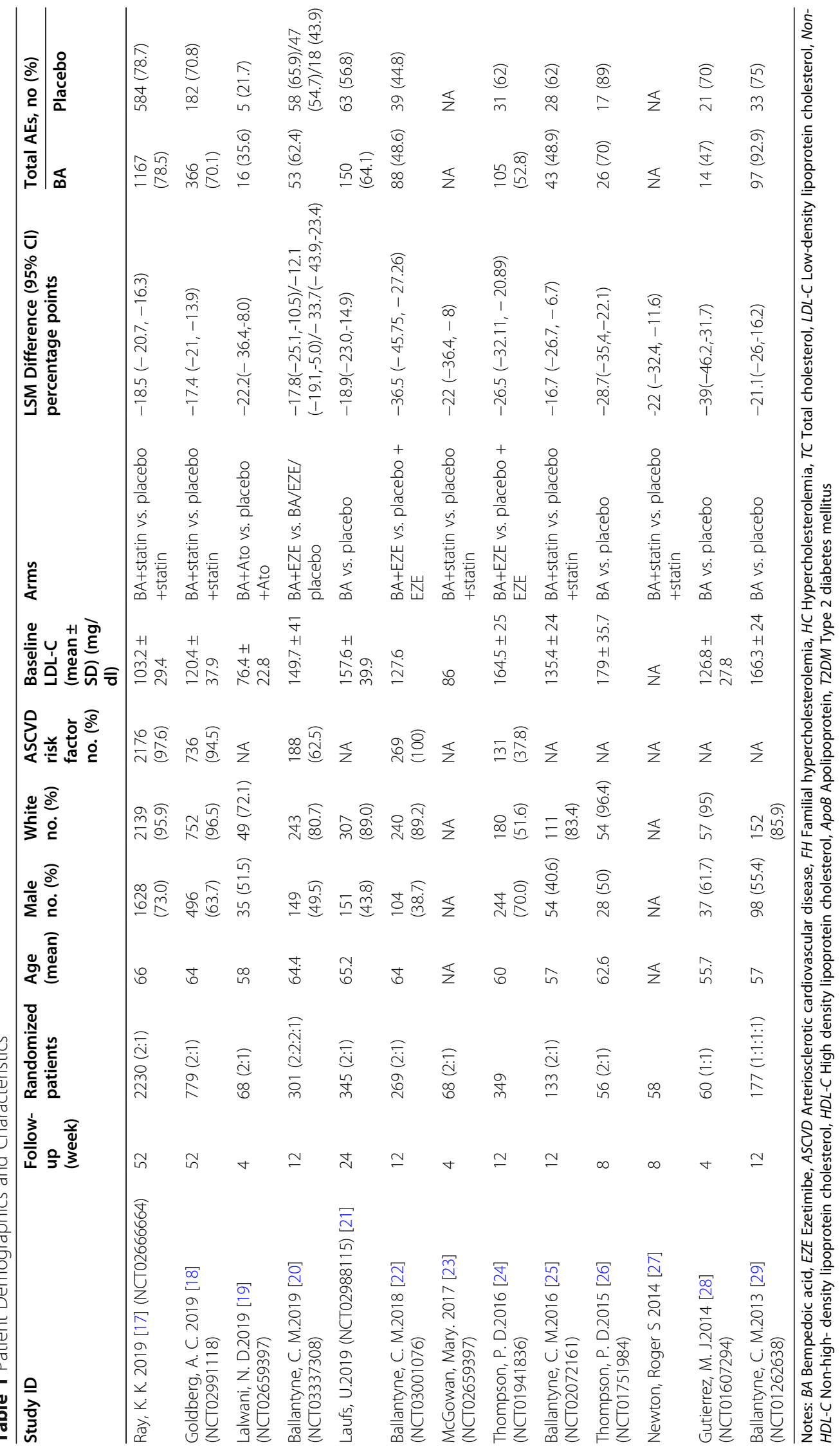




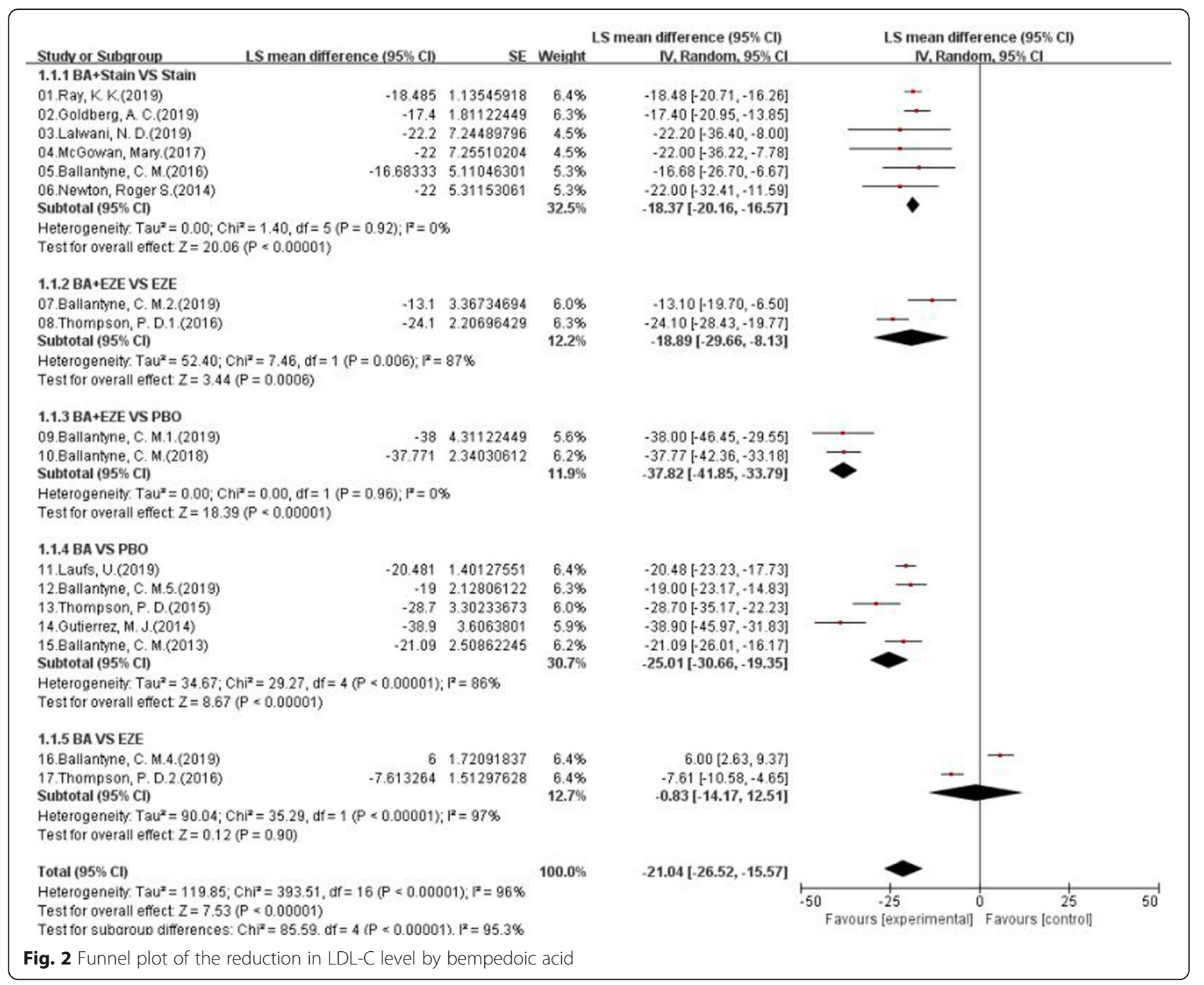

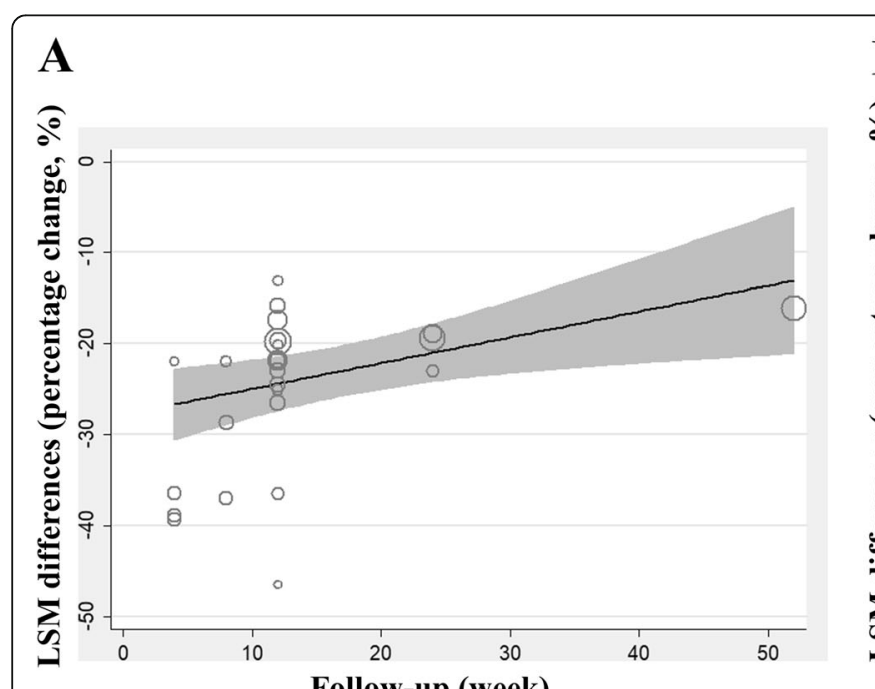

Follow-un (week)

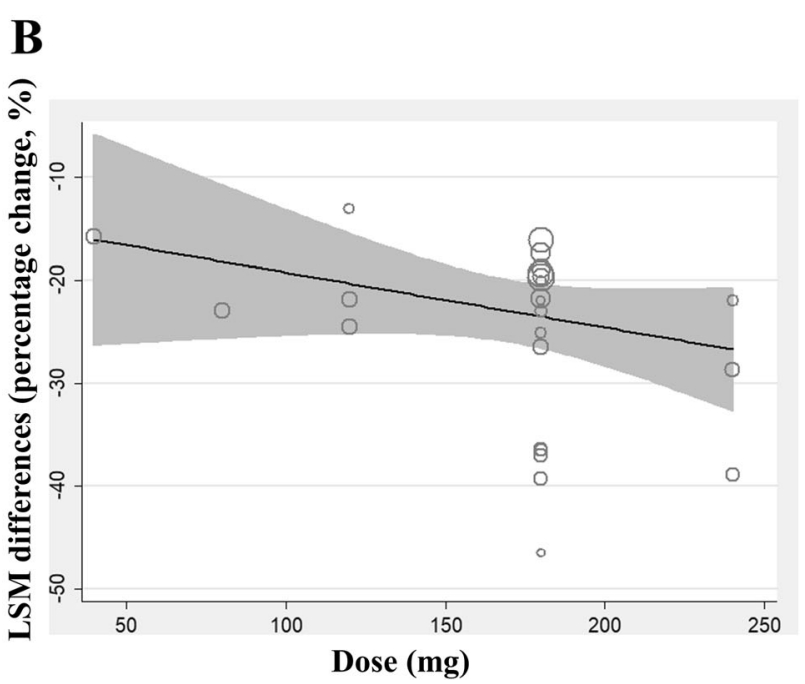

Fig. 3 Meta-regression analyses of the influences of treatment duration (a) and dose (b) on the magnitude of LDL-C-lowering by bempedoic acid 


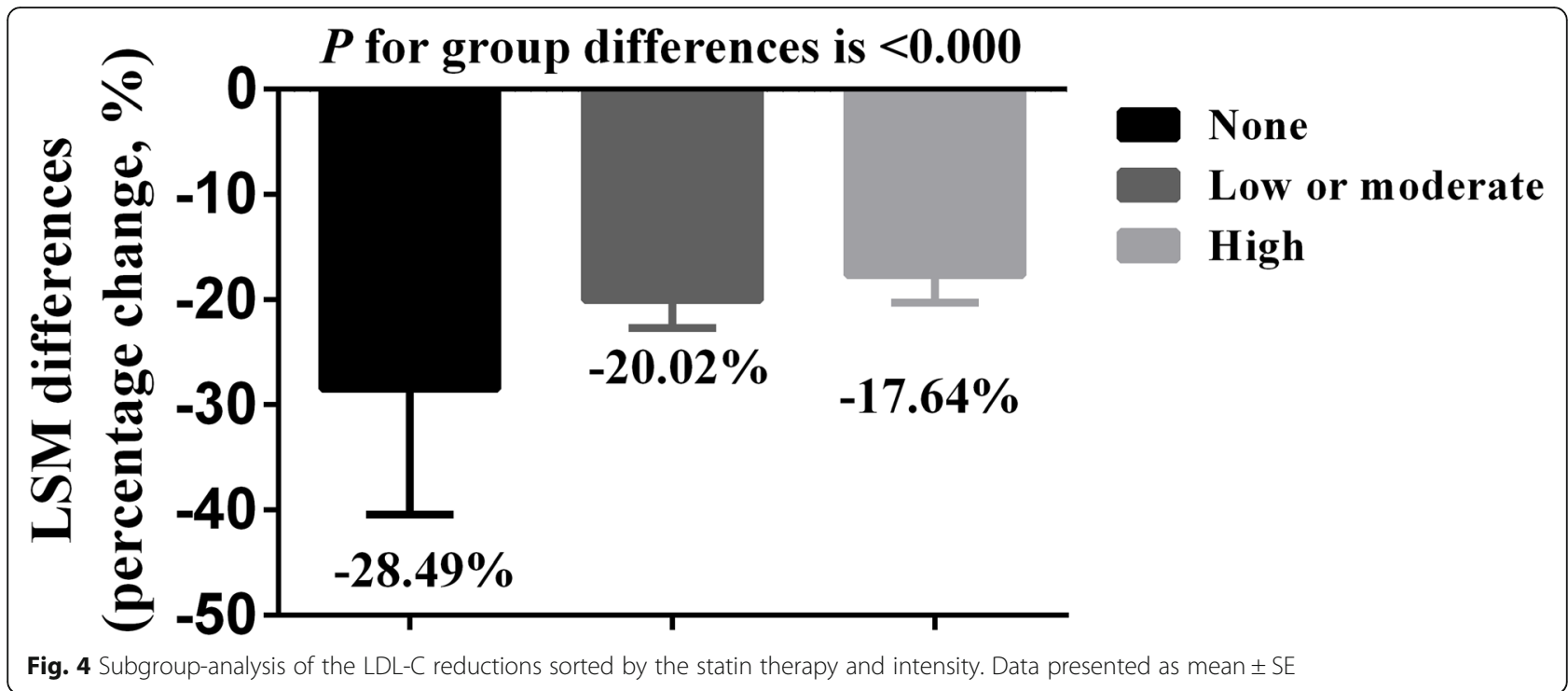

classified as "low risk of bias" in 13 included trials, allocation concealment in 7 trials and completeness of outcome data in 3 trials were classified as "unclear risk of bias". The issue of "anything else bias" was also hard to identified in the included trials. (Additional file 2). The potential publication bias of studies which regarded to the comparison of bempedoic acid plus statin versus statin alone was not obvious which was assessed by visual inspection of funnel plots for asymmetry (Additional file 3).

\section{Discussion}

In this study, pooled results showed that both bempedoic acid alone and combining with statin or ezetimibe all significantly reduced LDL-C level, the magnitudes of LDL-C reduction by the combination togethers (bempedoic acid

Table 2 Pooled results of TC, non-HDL-C, ApoB and hsCRP reductions by bempedoic acid

\begin{tabular}{|c|c|c|c|c|c|}
\hline \multirow[t]{2}{*}{ Subgroups } & \multirow{2}{*}{$\begin{array}{l}\text { Number } \\
\text { of studies }\end{array}$} & \multicolumn{2}{|l|}{ Sample size } & \multirow[t]{2}{*}{ ES $(95 \% \mathrm{Cl})$} & \multirow[t]{2}{*}{$I^{2}(\%)$} \\
\hline & & Experimental & Control & & \\
\hline \multicolumn{6}{|l|}{ TC } \\
\hline BA+Stain VS Stain & 4 & 2050 & 1045 & $-11.40(-12.15,-10.64)$ & 2.4 \\
\hline BA+EZE VS EZE & 1 & 86 & 86 & $-10.40(-16.15,-4.65)$ & - \\
\hline BA+EZE VS PBO & 1 & 86 & 41 & $-27.10(-35.10,-19.10)$ & - \\
\hline \multicolumn{6}{|l|}{ Non-HDL-C } \\
\hline BA+Stain VS Stain & 4 & 2050 & 1045 & $-13.57(-14.52,-12.62)$ & 0 \\
\hline BA+EZE VS EZE & 1 & 86 & 86 & $-12.10(-19.15,-5.05)$ & - \\
\hline $\mathrm{BA}+\mathrm{EZE}$ VS PBO & 1 & 86 & 41 & $-17.80(-25.10,-10.50)$ & - \\
\hline BA VS PBO & 1 & 224 & 107 & $-17.10(-20.50,-13.70)$ & - \\
\hline \multicolumn{6}{|l|}{ ApoB } \\
\hline BA+Stain VS Stain & 4 & 1998 & 1042 & $-12.73(-14.71,-10.75)$ & 45.8 \\
\hline BA+EZE VS EZE & 1 & 82 & 84 & $-9.30(-16.50 .-2.10)$ & - \\
\hline BA+EZE VS PBO & 1 & 82 & 38 & $-12.80(-20.30,-5.30)$ & - \\
\hline BA VS PBO & 1 & 224 & 107 & $-15.50(-18.80,-12.20)$ & - \\
\hline \multicolumn{6}{|l|}{ hsCRP } \\
\hline BA+Stain VS Stain & 4 & 2185 & 1109 & $-20.72(-29.21,-12.23)$ & 77.8 \\
\hline BA+EZE VS EZE & 1 & 80 & 79 & $-25.60(-44.50,-6.70)$ & - \\
\hline BA+EZE VS PBO & 1 & 80 & 39 & $-46.10(-77.60,-14.60)$ & - \\
\hline
\end{tabular}


Table 3 Pooled results of the incidence of AEs by bempedoic acid

\begin{tabular}{|c|c|c|c|c|c|c|c|}
\hline \multirow[t]{2}{*}{ Subgroup } & \multirow{2}{*}{$\begin{array}{l}\text { Number } \\
\text { of studies }\end{array}$} & \multicolumn{2}{|c|}{ Experimental } & \multicolumn{2}{|c|}{ Control } & \multirow[t]{2}{*}{ OR(95\% Cl) } & \multirow[t]{2}{*}{$r^{2}(\%)$} \\
\hline & & AEs & Total & AEs & Total & & \\
\hline \multicolumn{8}{|l|}{ Any AEs } \\
\hline BA+EZE VS EZE & 3 & 132 & 284 & 101 & 232 & $1.26(0.88,1.84)$ & 0 \\
\hline BA+Stain VS Stain & 4 & 1583 & 2109 & 795 & 1047 & $0.96(0.80,1.14)$ & 0 \\
\hline BA+EZE VS PBO & 1 & 13 & 53 & 4 & 18 & $1.14(0.32,4.07)$ & - \\
\hline BA VS PBO & 3 & 91 & 217 & 58 & 112 & $0.58(0.24,1.45)$ & 56.1 \\
\hline BA VS EZE & 1 & 105 & 198 & 53 & 98 & $0.96(0.59,1.56)$ & - \\
\hline \multicolumn{8}{|l|}{ Serious AEs } \\
\hline $\mathrm{BA}+\mathrm{EZE}$ VS EZE & 3 & 13 & 284 & 13 & 232 & $0.76(0.33,1.72)$ & 0 \\
\hline BA+Stain VS Stain & 4 & 323 & 2109 & 154 & 1047 & $1.05(0.85,1.30)$ & 0 \\
\hline BA+EZE VS PBO & 1 & 8 & 53 & 1 & 18 & $3.02(0.35,26.01)$ & - \\
\hline BA VS PBO & 2 & 15 & 187 & 4 & 82 & $1.53(0.52,4.53)$ & 0 \\
\hline BA VS EZE & 1 & 3 & 198 & 1 & 98 & $1.49(0.15,14.53)$ & - \\
\hline \multicolumn{8}{|c|}{ Discontinuation due to AEs } \\
\hline $\mathrm{BA}+\mathrm{EZE}$ VS EZE & 3 & 20 & 284 & 23 & 232 & $0.70(0.35,1.39)$ & 0 \\
\hline BA+Stain VS Stain & 3 & 165 & 1587 & 56 & 790 & $1.12(0.44,2.89)$ & 29.7 \\
\hline $\mathrm{BA}+\mathrm{EZE}$ VS PBO & 1 & 7 & 53 & 2 & 18 & $1.22(0.23,6.48)$ & - \\
\hline BA VS PBO & 2 & 43 & 187 & 16 & 82 & $0.44(0.02,9.81)$ & 76.3 \\
\hline BA VS EZE & 1 & 9 & 198 & 8 & 98 & $0.54(0.20,1.43)$ & - \\
\hline \multicolumn{8}{|l|}{ Muscle-related AEs } \\
\hline $\mathrm{BA}+\mathrm{EZE}$ VS EZE & 3 & 22 & 284 & 24 & 232 & $0.55(0.16,1.86)$ & 59.1 \\
\hline BA+Stain VS Stain & 4 & 226 & 2109 & 93 & 1047 & $1.29(1.00,1.67)^{a}$ & 0 \\
\hline BA+EZE VS PBO & 1 & 6 & 53 & 3 & 18 & $0.73(0.23,2.35)$ & - \\
\hline BA VS PBO & 2 & 40 & 187 & 24 & 82 & $0.66(0.37,1.20)$ & 0 \\
\hline BA VS EZE & 1 & 14 & 198 & 12 & 98 & $0.55(0.24,1.23)$ & - \\
\hline
\end{tabular}

Notes: $A E$ Adverse event, $B A$ Bempedoic acid, EZE Ezetimibe, $P B O$ Placebo. ${ }^{a}$, statistical significant

plus statin or ezetimibe) were similar but all stronger than these drugs alone. The treatment duration and the background of statin therapy may be the potential factors that influence the efficacy of bempedoic acid. In addition, bempedoic acid also lead to significant reductions in TC, nonHDL-C, ApoB and hsCRP levels. Importantly, current data showed the combination of bempedoic acid and statin may represent a trend of higher risk of muscle-related disorders when compared with statin alone.

Bempedoic acid, an oral inhibitor, is anticipated to lower the LDL-C level trough blocking ACL [7]. This metaanalysis confirmed that bempedoic acid significantly reduced the LDL-C level, which was consistent with the prior meta-analyses [10-12]. Of note, subgroup-analysis showed that the magnitude of reduction in LDL-C level varied largely among the different types of interventions. Bempedoic acid monotherapy presented the similar efficacy as ezetimibe. Because most participates had a history of statin intolerance before participated in trial, so no trial particularly compared the efficacy of bempedoic acid alone with statin alone. The efficacy of combination togethers (bempedoic acid + statin and bempedoic acid+ ezetimibe) were all stronger than statin or ezetimibe alone. Mechanism action of these agents may interpret the notion of "together is better". These results support the role of the combinations of bempedoic acid and statin or ezetimibe as an optional lipid-lowering strategy in patients with statin intolerance.

A phenomenon called "trail fatigue" is very common in many drugs studies. It is important to confirm whether the efficacy could be sustained with the extended period of treatment. Current data showed a slight attenuation of LDL-C reduction by bempedoic acid across week 4 to 52 of treatment. Such association needed to be explored in trials with longer-term exposure of bempedoic acid. Bempedoic acid works through the same cholesterol synthesis pathway as statins, it is interesting that whether statin therapy before screening could influence the efficacy of bempedoic acid. We observed the magnitude of reduction in LDL-C level decreased slightly as the growing intensity of statin therapy, but the net change point by bempedoic acid 
was also obvious even in the patients who have received high intensity of statin therapy, suggesting that bempedoic acid is an alternative for the patients with statin intolerance.

In addition, results showed that bempedoic acid also significantly reduced the other bad lipids level, including TC, non-HDL-C and ApoB, suggesting that bempedoic acid presents an additional effect on lipids level. High hsCRP in patients is closely related to the high risk of cardiovascular events. Clinical trials have demonstrated that patients with high hsCRP at baseline would get the most benefit from the lipid-lowering therapy with statin or PCSK9 inhibitors $[30,31]$. Results based on the current data showed that bempedoic acid substantially reduced hsCPR level, indicating that bempedoic acid may result in a benefit for patients in addition to lower lipids.

For the safety analysis, observed differences in any AEs, serious AEs and AEs which lead to discontinuation of treatment were not significant. For the statin-intolerant patients with hypercholesterolemia, clinical guidelines recommend combination therapy with statin and other nonstatin lipid-lowering drugs to reach the target of LDL-C level. Reducing the muscle-related disorders is the major concern for the combinative administration. Particularly, the liver-specific effect of bempedoic acid may resulted in reduced risk in muscle disorders as compared with statins. However, pooled results from this study showed a trend of higher risk in muscle-related AEs by the combination of bempedoic acid and statin when compared with statin monotherapy. Though it is not statistically significant, such risk need to be confirmed with more trials, because it is important for us to identify whether the combination of bempedoic acid and statin is safe for clinical practice. However, we did not compare the differences of muscle disorders risk between bempedoic acid alone and stain alone, which is a limitation in evaluating the safety of bempedoic.

Some limitations of this study listed are as follows. First, this study is a trial-level pooled analysis, it is hard to avoid potential bias. Second, though the efficacy of LDL-C reduction has been confirmed, this study failed to identify the cardiovascular risk reduction by bempedoic acid, one trial (CLEAR Outcomes, NCT02993406) is ongoing to assess the absolute CVD benefit of bempedoic acid. Third, the follow-up duration of completed trials was not long enough to observe the occurrence of AEs, which makes it difficult to fully confirm the safety of bempedoic aicd.

\section{Conclusions}

This study showed the efficacy of combination togethers (bempedoic acid plus statin or ezetimibe) were similar but all stronger than these drugs alone. Of note, a trend of high risk in muscle-related AEs by the combination of bempedoic acid and statin were observed, though it is not statistically significant, such risk was needed to be confirmed by more trials, because it is important for us to determine which is the better combinative administration for statin-intolerant patients. Now the absolute cardiovascular benefit of patients from bempedoic acid treatment is unclear, we anticipate the cardiovascular outcomes trials with bempedoic acid give us the answers.

\section{Supplementary Information}

The online version contains supplementary material available at https://doi. org/10.1186/s40360-020-00463-w.

Additional file 1. Search algorithm from Medline.

Additional file 2. Risk of bias in the included trials as assessed by the Cochrane risk of bias assessment tool.

Additional file 3. Publication bias assessment of included trials in the efficacy analysis.

\section{Abbreviations}

LDL-C: Low-density lipoprotein cholesterol; CVDs: Cardiovascular diseases; AEs: Adverse events; ACL: Adenosine triphosphate-citrate lyase; HMGCR: 3hydroxy-3-methylglutaryl-coenzyme A reductase; LDLR: LDL receptor; ACSV L1: Long-chain acyl-CoA synthetase 1; RCTs: Randomized control trials; TC: Total cholesterol; Non-HDL-C: Non-high-density lipoprotein cholesterol; ApoB: Apolipoprotein; hsCRP: High sensitivity C reactive protein; LSM: Least squares mean; WMD: Weight mean difference; Cl: Confidence interval; RRs: Risk rations

\section{Acknowledgements \\ Not applicable.}

\section{Authors' contributions}

QCF conceived and designed the research, ZX acquired the data, performed statistical analysis, drafted and approved the manuscript submitted. MXB performed statistical analysis, SZH and DZW carried out study search, JYX and Xiao ZP and SYL extracted data, LX and Jiang SL conducted quality assessment; LPF revised the manuscript, TLM and TB performed some data analysis. The authors read and approved the final manuscript.

\section{Funding}

This study was supported by the Natural Science Foundation of Hunan Province, China (2019JJ40230 and 2020JJ4072) and Project of Science and Technology of Health Commission of Hunan Province, China (B2019034 and B2019035). The funding bodies played no role in the design of the study and collection, analysis, and interpretation of data and in writing the manuscript.

\section{Availability of data and materials}

Data are available from the authors on request.

Ethics approval and consent to participate Not applicable.

\section{Consent for publication}

Not applicable.

\section{Competing interests}

The authors declare that they have no competing interests.

\section{Author details}

'Department of General Practice, The First People's Hospital of Huaihua, University of South China, Huaihua 418000, People's Republic of China. 2Evidence-based Medicine and Clinical Center, The First People's Hospital of Huaihua, University of South China, Huaihua 418000, People's Republic of 
China. ${ }^{3}$ Department of Pharmacology, The First People's Hospital of Huaihua, University of South China, Huaihua 418000, People's Republic of China. ${ }^{4}$ Department of Pharmacology, The Second People's Hospital of Huaihua City, Huaihua 418000, People's Republic of China. ${ }^{5}$ Department of Clinical Pharmacology, Xiangya Hospital, Central South University, Changsha 410008, People's Republic of China.

Received: 17 March 2020 Accepted: 19 November 2020

Published online: 04 December 2020

\section{References}

1. Ference BA, Ginsberg HN, Graham I, Ray KK, Packard CJ, Bruckert E, et al. Low-density lipoproteins cause atherosclerotic cardiovascular disease. 1. Evidence from genetic, epidemiologic, and clinical studies. A consensus statement from the European atherosclerosis society consensus panel. Eur Heart J. 2017;38:2459-72

2. Lloyd-Jones DM, Morris PB, Ballantyne CM, Birtcher KK, Daly DD Jr, DePalma SM, et al. 2017 focused update of the 2016 ACC expert consensus decision pathway on the role of non-statin therapies for LDL-cholesterol lowering in the Management of Atherosclerotic Cardiovascular Disease Risk: a report of the American College of Cardiology Task Force on expert consensus decision pathways. J Am Coll Cardiol. 2017;70:1785-822.

3. Silverman MG, Ference BA, Im K, Wiviott SD, Giugliano RP, Grundy SM, Braunwald $E$ and Sabatine MS. Association between lowering LDL-C and cardiovascular risk reduction among different therapeutic interventions: A systematic review and meta-analysis. Jama. 2016;316:1289-97.

4. Pinkosky SL, Newton RS, Day EA, Ford RJ, Lhotak S, Austin RC, et al. Liverspecific ATP-citrate lyase inhibition by bempedoic acid decreases LDL-C and attenuates atherosclerosis. Nat Commun. 2016;7:13457.

5. Ference BA, Ray KK, Catapano AL, Ference TB, Burgess S, Neff DR, et al. Mendelian randomization study of ACLY and cardiovascular disease. N Engl J Med. 2019;380:1033-42.

6. Ference BA. How to use Mendelian randomization to anticipate the results of randomized trials. Eur Heart J. 2018:39:360-2.

7. Saeed A, Ballantyne CM. Bempedoic acid (ETC-1002): a current review. Cardiol Clin. 2018;36:257-64.

8. Adhyaru BB, Jacobson TA. Safety and efficacy of statin therapy. Nat Rev Cardiol. 2018;15:757-69.

9. Bouitbir J, Sanvee GM, Panajatovic MV, Singh F and Krähenbühl S. Mechanisms of statin-associated skeletal muscle-associated symptoms. Pharmacol Res. 2020;154:104201.

10. Fernandes AFG, Knijnik LM, Maza MR, Penalver J, Cardoso R, Alfonso CE, et al. A meta-analysis of bempedoic acid for dyslipidemia in statin-intolerant patients. J Am Coll Cardiol. 2020;75:1933.

11. Wang X, Luo S, Gan X, He C, Huang R. Safety and efficacy of ETC-1002 in hypercholesterolaemic patients: a meta-analysis of randomised controlled trials. Kardiol Pol. 2019;77:207-16.

12. KSU KMU, Khan M, Lone AN, Valavoor S, Raghu C, Arshad A, et al. Meta analysis of efficacy and safety of bempedoic acid in lowering low density lipoprotein cholesterol. Circulation. 2019;140 supplement:1.13907.

13. Higgins JJGS. Cochrane handbook for systematic reviews of interventions. Version 5.1.0 [updated march 2011]: The Cochrane Collaboration; 2011. Available from www.cochrane-handbook.org.2011.

14. Qiu C, Zhao X, She L, Shi Z, Deng Z, Tan L, et al. Baricitinib induces LDL-C and HDL-C increases in rheumatoid arthritis: a meta-analysis of randomized controlled trials. Lipids Health Dis. 2019;18:54.

15. Higgins JP, Thompson SG. Quantifying heterogeneity in a meta-analysis. Stat Med. 2002;21:1539-58.

16. Egger M, Davey Smith G, Schneider M, Minder C. Bias in meta-analysis detected by a simple, graphical test. Bmj. 1997;315:629-34.

17. Ray KK, Bays HE, Catapano AL, Lalwani ND, Bloedon LT, Sterling LR, et al. Safety and efficacy of Bempedoic acid to reduce LDL cholesterol. N Engl J Med. 2019;380:1022-32.

18. Goldberg AC, Leiter LA, Stroes ESG, Baum SJ, Hanselman JC, Bloedon LT, et al. Effect of Bempedoic acid vs placebo added to maximally tolerated statins on low-density lipoprotein cholesterol in patients at high risk for cardiovascular disease: the CLEAR wisdom randomized clinical trial. Jama. 2019:322:1780-8.

19. Lalwani ND, Hanselman JC, MacDougall DE, Sterling LR, Cramer CT. Complementary low-density lipoprotein-cholesterol lowering and pharmacokinetics of adding bempedoic acid (ETC-1002) to high-dose atorvastatin background therapy in hypercholesterolemic patients: a randomized placebo-controlled trial. J Clin Lipidol. 2019;13:568-79.

20. Ballantyne C, Laufs U, Ray KK, Leiter LA, Bays HE, Goldberg AC, et al. Efficacy and safety of Bempedoic acid + Ezetimibe fixed-dose combination in patients at high CVD risk and with elevated LDL-C receiving maximally tolerated statin therapy. Atherosclerosis. 2019;287:e7-8.

21. Laufs U, Banach M, Mancini GBJ, Gaudet D, Bloedon LT, Sterling LR, et al. Efficacy and safety of Bempedoic acid in patients with hypercholesterolemia and statin intolerance. J Am Heart Assoc. 2019;8:e011662.

22. Ballantyne CM, Banach M, Mancini GBJ, Lepor NE, Hanselman JC, Zhao X, et al. Efficacy and safety of bempedoic acid added to ezetimibe in statinintolerant patients with hypercholesterolemia: a randomized, placebocontrolled study. Atherosclerosis. 2018;277:195-203.

23. McGowan M, MacDougall D, Hanselman J, Watling S and Lalwani N. Bempedoic acid reduces $L D L-C$ and is well-Tolerated in patients receiving atorvastatin $80 \mathrm{mg}$ background therapy. J Clin Lipidol. 2017;11:838.

24. Thompson PD, MacDougall DE, Newton RS, Margulies JR, Hanselman JC, Orloff DG, et al. Treatment with ETC-1002 alone and in combination with ezetimibe lowers LDL cholesterol in hypercholesterolemic patients with or without statin intolerance. J Clin Lipidol. 2016;10:556-67.

25. Ballantyne $C M$, McKenney JM, MacDougall $D E$, Margulies JR, Robinson PL, Hanselman JC, et al. Effect of ETC-1002 on serum low-density lipoprotein cholesterol in Hypercholesterolemic patients receiving statin therapy. Am J Cardiol. 2016;117:1928-33.

26. Thompson PD, Rubino J, Janik MJ, MacDougall DE, McBride SJ, Margulies JR, et al. Use of ETC-1002 to treat hypercholesterolemia in patients with statin intolerance. J Clin Lipidol. 2015;9:295-304.

27. Newton RS, Ballantyne CM, Thompson PD, MacDougall DE, Hanselman JC, Margulies JR, et al. ETC-1002 lowers LDL-cholesterol and is well tolerated in Hypercholesterolemic patients across four phase 2a studies. J Clin Lipidol. 2014;8:339-40.

28. Gutierrez MJ, Rosenberg NL, Macdougall DE, Hanselman JC, Margulies JR, Strange P, et al. Efficacy and safety of ETC-1002, a novel investigational lowdensity lipoprotein-cholesterol-lowering therapy for the treatment of patients with hypercholesterolemia and type 2 diabetes mellitus. Arterioscler Thromb Vasc Biol. 2014;34:676-83.

29. Ballantyne CM, Davidson MH, Macdougall DE, Bays HE, Dicarlo LA Rosenberg NL, et al. Efficacy and safety of a novel dual modulator of adenosine triphosphate-citrate lyase and adenosine monophosphateactivated protein kinase in patients with hypercholesterolemia: results of a multicenter, randomized, double-blind, placebo-controlled, parallel-group trial. J Am Coll Cardiol. 2013;62:1154-62.

30. Tabrizi R, Tamtaji OR, Mirhosseini N, Lankarani KB, Akbari M, Dadgostar E, et al. The effects of statin use on inflammatory markers among patients with metabolic syndrome and related disorders: a systematic review and metaanalysis of randomized controlled trials. Pharmacol Res. 2019;141:85-103.

31. Tunon J, Badimon L, Bochaton-Piallat ML, Cariou B, Daemen MJ, Egido J, et al. Identifying the anti-inflammatory response to lipid lowering therapy: a position paper from the working group on atherosclerosis and vascular biology of the European Society of Cardiology. Cardiovasc Res. 2019;115:10-9.

\section{Publisher's Note}

Springer Nature remains neutral with regard to jurisdictional claims in published maps and institutional affiliations.

Ready to submit your research? Choose BMC and benefit from:

- fast, convenient online submission

- thorough peer review by experienced researchers in your field

- rapid publication on acceptance

- support for research data, including large and complex data types

- gold Open Access which fosters wider collaboration and increased citations

- maximum visibility for your research: over $100 \mathrm{M}$ website views per year

At BMC, research is always in progress.

Learn more biomedcentral.com/submission 